\title{
Acute Coronary Syndrome
}

National Cancer Institute

\section{Source}

National Cancer Institute. Acute Coronary Syndrome. NCI Thesaurus. Code C53652.

Signs and symptoms related to acute ischemia of the myocardium secondary to coronary artery disease. The clinical presentation covers a spectrum of heart diseases from unstable angina to myocardial infarction. 